\title{
Approximation properties of weighted Kantorovich type operators in compact disks
}

\section{Nazim I Mahmudov and Mustafa Kara*}

\section{"Correspondence:}

mustafa.kara@emu.edu.tr

Eastern Mediterranean University,

Mersin 10, Gazimagusa, TRNC,

Turkey

\begin{abstract}
In this paper, we discuss the approximation properties of the complex weighted Kantorovich type operators. Quantitative estimates of the convergence, the Voronovskaja type theorem, and saturation of convergence for complex weighted Kantorovich polynomials attached to analytic functions in compact disks will be given. In particular, we show that for functions analytic in $\{z \in C:|z|<R\}$, the rate of approximation by the weighted complex Kantorovich type operators is $1 / n$.
\end{abstract}

Keywords: weighted Bernstein-Kantorovich operators; Bernstein-Kantorovich operator; complex approximation

\section{Introduction}

The first constructive (and simple) proof of Weierstrass approximation theorem was given by Bernstein [1]. He gave an alternative proof to the Weierstrass approximation theorem and introduced the following polynomial:

$$
B_{n}(f)(x)=\sum_{k=0}^{n} p_{n, k}(x) f\left(\frac{k}{n}\right), \quad f:[0,1] \rightarrow \mathbb{R}
$$

where $p_{n, k}(x)=\left(\begin{array}{l}n \\ k\end{array}\right) x^{k}(1-x)^{n-k}, x \in[0,1]$.

For any $f \in L^{p}([0,1]), 1 \leq p \leq \infty$, Ditzian and Totik (see [2]) introduced the KantorovichBernstein operator as follows:

$$
K_{n}(f ; x)=\sum_{k=0}^{n} p_{n, k}(x)(n+1) \int_{\frac{k+1}{n+1}}^{\frac{k}{n+1}} f(u) d u,
$$

where $p_{n, k}(x)=\left(\begin{array}{l}n \\ k\end{array}\right) x^{k}(1-x)^{n-k}, k=0,1,2, \ldots, n ; x \in[0,1]$.

Let

$$
w(x)=x^{\alpha}(1-x)^{\beta}, \quad \alpha, \beta>-1,0 \leq x \leq 1,
$$

be the classical Jacobi weights.

Let

$$
L_{w}^{p}= \begin{cases}\left\{f: w f \in L^{p}(0,1)\right\}, & 1 \leq p<\infty, \\ \left\{f: f \in(0,1), \lim _{x(1-x) \rightarrow 0}(w f)(x)=0\right\}, & p=\infty,\end{cases}
$$

(C) 2015 Mahmudov and Kara; licensee Springer. This is an Open Access article distributed under the terms of the Creative Commons Attribution License (http://creativecommons.org/licenses/by/4.0), which permits unrestricted use, distribution, and reproduction in any medium, provided the original work is properly credited. 
and equip $L_{w}^{p}$ with norm

$$
\|w f\|_{p}= \begin{cases}\left(\int_{0}^{1}|(w f)(x)|^{p} d x\right)^{1 / p}, & 1 \leq p<\infty, \\ \sup _{0 \leq x \leq 1}|(w f)(x)|, & p=\infty .\end{cases}
$$

In [2], Ditzian and Totik studied the case of weighted approximation properties of $K_{n}(f ; x)$ in $L_{w}^{p}$ under the restrictions $-\frac{1}{p}<\alpha, \beta<1-\frac{1}{p}$ on the weighted parameters. In [3], Della Vecchia et al. removed the restrictions on $\alpha, \beta$ and introduced a weighted generalization of the $K_{n}(f ; x)$ as follows:

$$
K_{n}^{*}(f ; x)=\sum_{k=0}^{n} \frac{\int_{I_{k}}(w f)(t) d t}{\int_{I_{k}} w(t) d t} p_{n, k}(x), \quad x \in[0,1],
$$

where

$$
-\frac{1}{p}<\alpha, \beta, \quad 1 \leq p \leq \infty
$$

and $f \in L_{w}^{p}$. $K_{n}^{*}(f ; x)$ allows a wider class of functions than the operator $K_{n}(f ; x)$. Because, Della Vecchia et al. dropped the restrictions $\alpha, \beta<1-\frac{1}{p}$ and obtained (see [3]) direct and converse theorems and a Voronovskaya-type relation for the weighted Kantorovich operator (3). Also Della Vecchia et al. solved the saturation problem of the weighted Kantorovich operator (3) (see [4, Theorem 2.1]).

In [5], Yu introduced the following modified operator of $K_{n}(f ; x)$ :

$$
\begin{aligned}
K_{n}^{*}(f ; x):= & (1-x)^{n}(n+1)\left(2 \int_{I_{1}} f(t) d t-\int_{I_{2}} f(t) d t\right) \\
& +\sum_{k=1}^{n-1}(n+1) \int_{I_{k}} f(u) d u p_{n k}(x) \\
& +x^{n}(n+1)\left(2 \int_{I_{n-1}} f(t) d t-\int_{I_{n-2}} f(t) d t\right),
\end{aligned}
$$

and direct and converse theorems and a Voronovskaya-type relation were obtained with modification of the classical Kantorovich-Bernstein operators (1) with Jacobi weights $w(x)=x^{\alpha}(1-x)^{\beta}$, where $-\frac{1}{p}<\alpha, \beta$.

The goal of the present note is to introduce the complex weighted Kantorovich type operator

$$
K_{n}(f ; z)=\sum_{k=0}^{n} p_{n, k}(z) \frac{\int_{0}^{1} t^{\alpha}(1-t)^{\beta} f\left(\frac{k+t}{n+1}\right) d t}{B(\alpha+1, \beta+1)},
$$

where $\frac{-1}{p}<\alpha, \beta$ and $1 \leq p \leq \infty, B(\cdot, \cdot)$ is a beta function, and we study the convergence properties of $K_{n}(f ; z)$. Notice that the approximation properties of complex generalized Kantorovich type operators are studied in [6].

In the recent books of Gal $[7,8]$ (see references therein), a systematic study of the overconvergence phenomenon in a complex approximation was made for the following important classes of Bernstein-type operators: Bernstein, Bernstein-Faber, Bernstein-Butzer, 
Bernstein-Stancu, Bernstein-Kantorovich, Favard-Szasz-Mirakjan, Baskakov, BernsteinDurrmeyer, and Balazs-Szabados.

Let $\mathbb{D}_{R}$ be a disc $\mathbb{D}_{R}:=\{z \in \mathbb{C}:|z|<R\}$ in the complex plane $\mathbb{C}$. Denote by $H\left(\mathbb{D}_{R}\right)$ the space of all analytic functions on $\mathbb{D}_{R}$. For $f \in H\left(\mathbb{D}_{R}\right)$ we assume that $f(z)=\sum_{m=0}^{\infty} a_{m} z^{m}$.

We start with the following quantitative estimates of the convergence for complex weighted Kantorovich type operators attached to an analytic function in a disk of radius $R>1$ and center 0 .

Theorem 1 Let $f \in H\left(\mathbb{D}_{R}\right)$. If $1 \leq r<R$, then for all $|z| \leq r$ we have

$$
\left|K_{n}(f ; z)-f(z)\right| \leq \frac{2}{n} \sum_{m=1}^{\infty}\left|c_{m}\right| m(m+1) r^{m}
$$

where $n \in \mathbb{N}$.

The next theorem gives a Voronovskaja type result in compact disks, for complex weighted Kantorovich type operators attached to an analytic function in $\mathbb{D}_{R}$, where $R>1$, and with center 0 .

Theorem 2 Let $f \in H\left(\mathbb{D}_{R}\right)$. If $1 \leq r<R$ then, for all $|z| \leq r$, we have

$$
\begin{aligned}
& \left|K_{n}(f ; z)-f(z)-\frac{(\alpha+1)-z(\alpha+\beta+2)}{(\alpha+\beta+2)(n+1)} f^{\prime}(z)-\frac{z(1-z)}{2(n+1)} f^{\prime \prime}(z)\right| \\
& \quad \leq \frac{13}{n^{2}} \sum_{m=2}^{\infty}\left|a_{m}\right| m(m-1)^{2} r^{m}+\frac{4}{(\alpha+\beta+2) n^{2}} \sum_{m=3}^{\infty}\left|a_{m}\right| m(m-1) r^{m},
\end{aligned}
$$

where $n \in \mathbb{N}$.

As an application of Theorem 2 we present the order of approximation for complex weighted Kantorovich type operators.

Theorem 3 Let $f \in H\left(\mathbb{D}_{R}\right)$.If $1 \leq r<R$ and iff is not a constant function, then the estimate

$$
\left\|K_{n}(f)-f\right\|_{r} \geq \frac{1}{n} C_{r}(f), \quad n \in \mathbb{N}
$$

holds, where the constant $C_{r}(f)$ depends on $f, \alpha, \beta$, and $r$ but it is independent of $n$.

\section{Auxiliary results}

Lemma 4 For all $n \in \mathbb{N}, m \in \mathbb{N} \cup\{0\}, z \in \mathbb{C}$ we have

$$
K_{n}\left(e_{m} ; z\right)=\frac{1}{(n+1)^{m}} \sum_{j=0}^{m}\left(\begin{array}{c}
m \\
j
\end{array}\right) \frac{n^{j} B(\alpha+m-j+1, \beta+1)}{B(\alpha+1, \beta+1)} B_{n}\left(e_{j} ; z\right),
$$

where $e_{m}(z)=z^{m}$. 
Proof The recurrence formula can be derived by direct computation:

$$
\begin{aligned}
K_{n}\left(e_{m} ; z\right) & =\frac{1}{(n+1)^{m}} \sum_{k=0}^{n} p_{n, k}(z) \frac{1}{B(\alpha+1, \beta+1)} \sum_{j=0}^{m} \int_{0}^{1}\left(\begin{array}{c}
m \\
j
\end{array}\right) t^{\alpha}(1-t)^{\beta} k^{j} t^{m-j} d t \\
& =\frac{1}{(n+1)^{m}} \sum_{k=0}^{n} p_{n, k}(z) \frac{1}{B(\alpha+1, \beta+1)} \sum_{j=0}^{m}\left(\begin{array}{c}
m \\
j
\end{array}\right) k^{j} \int_{0}^{1} t^{\alpha+m-j}(1-t)^{\beta} d t \\
& =\frac{1}{(n+1)^{m}} \sum_{j=0}^{m}\left(\begin{array}{c}
m \\
j
\end{array}\right) \frac{n^{j} B(\alpha+m-j+1, \beta+1)}{B(\alpha+1, \beta+1)} B_{n}\left(e_{j} ; z\right) .
\end{aligned}
$$

Lemma 5 We have

$$
\begin{aligned}
& K_{n}\left(e_{0} ; z\right)=1, \\
& \begin{aligned}
K_{n}\left(e_{1} ; z\right)= & \frac{\alpha+1}{(\alpha+\beta+2)(n+1)}+\frac{n z}{n+1}, \\
K_{n}\left(e_{2} ; z\right)= & \frac{1}{(n+1)^{2}} \frac{(\alpha+2)(\alpha+1)}{(\alpha+\beta+3)(\alpha+\beta+2)}+\frac{2 n z}{(n+1)^{2}} \frac{(\alpha+1)}{(\alpha+\beta+2)} \\
& +\left(z^{2}+\frac{z(1-z)}{n}\right) \frac{n^{2}}{(n+1)^{2}}, \\
K_{n}\left(\left(e_{1}-z\right) ; z\right)= & \frac{(\alpha+1)-z(\alpha+\beta+2)}{(n+1)(\alpha+\beta+2)}, \\
K_{n}\left(\left(e_{1}-z\right)^{2} ; z\right)= & n^{2} \frac{z^{2}+\frac{1}{n} z(1-z)}{(n+1)^{2}}-z^{2}-2 z\left(\frac{\alpha+1}{(n+1)(\alpha+\beta+2)}+\frac{n z}{n+1}\right) \\
& +2 n z \frac{\alpha+1}{(n+1)^{2}(\alpha+\beta+2)}+\frac{(\alpha+1)(\alpha+2)}{(n+1)^{2}(\alpha+\beta+2)(\alpha+\beta+3)} .
\end{aligned}
\end{aligned}
$$

Lemma 6 For all $z \in \mathbb{D}_{r}, r \geq 1$ we have

$$
\left|K_{n}\left(e_{m} ; z\right)\right| \leq r^{m}
$$

where $e_{m}(z)=z^{m}$.

Proof Indeed, using the inequality $\left|B_{n}\left(e_{j} ; z\right)\right| \leq r^{j}$ (see [7]) we get

$$
\begin{aligned}
\left|K_{n}\left(e_{m} ; z\right)\right| & \leq \sum_{j=0}^{m}\left(\begin{array}{c}
m \\
j
\end{array}\right) \frac{n^{j} B(\alpha+m-j+1, \beta+1)}{(n+1)^{m} B(\alpha+1, \beta+1)}\left|B_{n}\left(e_{j} ; z\right)\right| \\
& \leq \frac{1}{(n+1)^{m}} \sum_{j=0}^{m}\left(\begin{array}{c}
m \\
j
\end{array}\right) n^{j} r^{m}=\left(\frac{1+n}{n+1}\right)^{m} r^{m}=r^{m} .
\end{aligned}
$$

Lemma 7 For all $z \in \mathbb{C}, z \in \mathbb{N} \cup\{0\}$ we have

$$
\begin{aligned}
K_{n}\left(e_{m+1} ; z\right)= & \frac{z(1-z)}{n} K_{n}\left(e_{m} ; z\right)+z K_{n}\left(e_{m} ; z\right) \\
& +\frac{1}{(n+1)^{m}} \sum_{j=0}^{m+1}\left(\begin{array}{c}
m+1 \\
j
\end{array}\right) n^{j-1} \frac{B(\alpha+m-j+2, \beta+1)}{B(\alpha+1, \beta+1)} \\
& \times\left(\frac{n}{n+1}-\frac{j}{m+1}\right) B_{n}\left(e_{j} ; z\right) .
\end{aligned}
$$


Proof We know that (see [7])

$$
\frac{z(1-z)}{n} B_{n}^{\prime}\left(e_{j} ; z\right)=B_{n}\left(e_{j+1} ; z\right)-z B_{n}\left(e_{j} ; z\right)
$$

Taking the derivative of (10) and using the above formula we have

$$
\begin{aligned}
\frac{z(1-z)}{n} K_{n}^{\prime}\left(e_{m} ; z\right) & =\frac{z(1-z)}{n} \sum_{j=0}^{m}\left(\begin{array}{c}
m \\
j
\end{array}\right) \frac{n^{j} B(\alpha+m-j+1, \beta+1)}{(n+1)^{m} B(\alpha+1, \beta+1)} B_{n}^{\prime}\left(e_{j} ; z\right) \\
& =\sum_{j=0}^{m}\left(\begin{array}{c}
m \\
j
\end{array}\right) \frac{n^{j} B(\alpha+m-j+1, \beta+1)}{(n+1)^{m} B(\alpha+1, \beta+1)}\left(B_{n}\left(e_{j+1} ; z\right)-z B_{n}\left(e_{j} ; z\right)\right) \\
& =\sum_{j=1}^{m+1}\left(\begin{array}{c}
m \\
j-1
\end{array}\right) \frac{n^{j-1} B(\alpha+m-j+2, \beta+1)}{(n+1)^{m} B(\alpha+1, \beta+1)} B_{n}\left(e_{j} ; z\right)-z K_{n}\left(e_{m} ; z\right) .
\end{aligned}
$$

It follows that

$$
\begin{aligned}
K_{n}\left(e_{m+1} ; z\right)= & \frac{z(1-z)}{n} K_{n}^{\prime}\left(e_{m} ; z\right)+z K_{n}\left(e_{m} ; z\right) \\
& +\sum_{j=0}^{m+1}\left(\begin{array}{c}
m+1 \\
j
\end{array}\right) \frac{n^{j} B(\alpha+m-j+2, \beta+1)}{(n+1)^{m+1} B(\alpha+1, \beta+1)} B_{n}\left(e_{j} ; z\right) \\
& -\sum_{j=1}^{m+1}\left(\begin{array}{c}
m \\
j-1
\end{array}\right) \frac{n^{j-1} B(\alpha+m-j+2, \beta+1)}{(n+1)^{m+1} B(\alpha+1, \beta+1)} B_{n}\left(e_{j} ; z\right) \\
= & \frac{z(1-z)}{n} K_{n}^{\prime}\left(e_{m} ; z\right)+z K_{n}\left(e_{m} ; z\right)+\frac{B(\alpha+m+2, \beta+1)}{(n+1)^{m+1} B(\alpha+1, \beta+1)} \\
& +\sum_{j=1}^{m+1}\left(\begin{array}{c}
m+1 \\
j
\end{array}\right) \frac{n^{j-1} B(\alpha+m-j+2, \beta+1)}{(n+1)^{m} B(\alpha+1, \beta+1)}\left(\frac{-j}{m+1}+\frac{n}{n+1}\right) B_{n}\left(e_{j} ; z\right) \\
= & \frac{z(1-z)}{n} K_{n}^{\prime}\left(e_{m} ; z\right)+z K_{n}\left(e_{m} ; z\right) \\
& +\sum_{j=0}^{m+1}\left(\begin{array}{c}
m+1 \\
j
\end{array}\right) \frac{n^{j-1} B(\alpha+m-j+2, \beta+1)}{(n+1)^{m} B(\alpha+1, \beta+1)}\left(\frac{-j}{m+1}+\frac{n}{n+1}\right) B_{n}\left(e_{j} ; z\right) .
\end{aligned}
$$

Here we used the identity

$$
\left(\begin{array}{c}
m \\
j-1
\end{array}\right)=\left(\begin{array}{c}
m+1 \\
j
\end{array}\right) \frac{j}{(m+1)}
$$

Define

$$
E_{n, m}(z):=K_{n}\left(e_{m} ; z\right)-e_{m}(z)-\frac{(\alpha+1)-z(\alpha+\beta+2)}{(\alpha+\beta+2)(n+1)} m z^{m-1}-\frac{z(1-z)}{2(n+1)} m(m-1) z^{m-2} .
$$

Lemma 8 Let $n, m \in \mathbb{N}$, we have the following recurrence formula:

$$
\begin{aligned}
E_{n, m}(z)= & \frac{z(1-z)}{n}\left(K_{n, q}\left(e_{m-1} ; z\right)-e_{m-1}(z)\right)^{\prime} \\
& +z E_{n, m-1}(z)-\frac{m-1}{n(n+1)} z^{m-1}(1-z)
\end{aligned}
$$




$$
\begin{aligned}
& -\frac{(\alpha+1)-z(\alpha+\beta+2)}{(\alpha+\beta+2)(n+1)} z^{m-1} \\
& +\frac{1}{(n+1)^{m}} \sum_{j=0}^{m}\left(\begin{array}{c}
m \\
j
\end{array}\right) \frac{n^{j} B(\alpha+m-j+1, \beta+1)}{B(\alpha+1, \beta+1)} \frac{m n-j(n+1)}{m n} B_{n}\left(e_{j} ; z\right) .
\end{aligned}
$$

Proof It is immediate that $E_{n, m}(z)$ is a polynomial of degree less than or equal to $m$ and that $E_{n, 0}(z)=E_{n, 1}(z)=0$.

Using (11) we get

$$
\begin{aligned}
& E_{n, m}(z)=\frac{z(1-z)}{n}\left(K_{n}\left(e_{m-1} ; z\right)-e_{m-1}(z)\right)^{\prime}+\frac{(m-1)}{n} z^{m-1}(1-z) \\
& +z\left(E_{n, m-1}(z)+\frac{(\alpha+1)-z(\alpha+\beta+2)}{(\alpha+\beta+2)(n+1)}(m-1) z^{m-2}\right. \\
& \left.+\frac{z(1-z)}{2(n+1)}(m-1)(m-2) z^{m-3}\right) \\
& -\frac{(\alpha+1)-z(\alpha+\beta+2)}{(\alpha+\beta+2)(n+1)} m z^{m-1}-\frac{z(1-z)}{2(n+1)} m(m-1) z^{m-2} \\
& +\frac{1}{(n+1)^{m}} \sum_{j=0}^{m}\left(\begin{array}{c}
m \\
j
\end{array}\right) \frac{n^{j} B(\alpha+m-j+1, \beta+1)}{B(\alpha+1, \beta+1)} \frac{m n-j(n+1)}{m n} B_{n}\left(e_{j} ; z\right), \\
& E_{n, m}(z)=\frac{z(1-z)}{n}\left(K_{n}\left(e_{m-1} ; z\right)-e_{m-1}(z)\right)^{\prime}+z^{m-1} \frac{(\alpha+1)-z(\alpha+\beta+2)}{(\alpha+\beta+2)(n+1)}(m-1-m) \\
& +z^{m-1}(1-z) \frac{(m-1)(m-2-m)}{2(n+1)}+z^{m-1}(1-z) \frac{(m-1)}{n} \\
& +\frac{1}{(n+1)^{m}} \sum_{j=0}^{m}\left(\begin{array}{c}
m \\
j
\end{array}\right) \frac{n^{j} B(\alpha+m-j+1, \beta+1)}{B(\alpha+1, \beta+1)} \frac{m n-j(n+1)}{m n} B_{n}\left(e_{j} ; z\right), \\
& E_{n, m}(z)=\frac{z(1-z)}{n}\left(K_{n, q}\left(e_{m-1} ; z\right)-e_{m-1}(z)\right)^{\prime} \\
& +z E_{n, m-1}(z)+\frac{(m-1)}{n(n+1)} z^{m-1}(1-z)-\frac{(\alpha+1)-z(\alpha+\beta+2)}{(\alpha+\beta+2)(n+1)} z^{m-1} \\
& +\frac{1}{(n+1)^{m}} \sum_{j=0}^{m}\left(\begin{array}{c}
m \\
j
\end{array}\right) \frac{n^{j} B(\alpha+m-j+1, \beta+1)}{B(\alpha+1, \beta+1)} \frac{m n-j(n+1)}{m n} B_{n}\left(e_{j} ; z\right),
\end{aligned}
$$

which is the desired recurrence formula.

\section{Proofs of the main results}

Proof of Theorem 1 By use of the above recurrence we obtain the following relationship:

$$
\begin{aligned}
K_{n}\left(e_{m} ; z\right)-e_{m}(z)= & \frac{z(1-z)}{n} K_{n}^{\prime}\left(e_{m-1} ; z\right)+z\left(K_{n}\left(e_{m-1} ; z\right)-e_{m-1}(z)\right) \\
& +\frac{1}{(n+1)^{m}} \sum_{j=0}^{m}\left(\begin{array}{c}
m \\
j
\end{array}\right) \frac{n^{j} B(\alpha+m-j+1, \beta+1)}{B(\alpha+1, \beta+1)} \\
& \times \frac{m n-j(n+1)}{m n} B_{n}\left(e_{j} ; z\right) .
\end{aligned}
$$


For $|z| \leq r$ we can easily estimate the sum in the above formula as follows:

$$
\begin{aligned}
\mid & \frac{1}{(n+1)^{m}} \sum_{j=0}^{m}\left(\begin{array}{c}
m \\
j
\end{array}\right) n^{j} \frac{B(\alpha+m-j+1, \beta+1)}{B(\alpha+1, \beta+1)}\left(1-\frac{j}{m}-\frac{j}{m n}\right) B_{n}\left(e_{j} ; z\right) \mid \\
\leq & \frac{1}{(n+1)^{m}}\left(\sum_{j=0}^{m-1}\left(\begin{array}{c}
m-1 \\
j
\end{array}\right) \frac{m}{m-j} \frac{n^{j} B(\alpha+m-j+1, \beta+1)}{B(\alpha+1, \beta+1)}\left|1-\frac{j}{m}-\frac{j}{m n}\right|\right)\left|B_{n}\left(e_{j} ; z\right)\right| \\
& +\frac{B(\alpha+1, \beta+1)}{B(\alpha+1, \beta+1)} \frac{n^{m-1}}{(n+1)^{m}} r^{m} \\
\leq & \frac{1}{(n+1)^{m}}\left(\sum_{j=0}^{m-1}\left(\begin{array}{c}
m-1 \\
j
\end{array}\right) \frac{m n^{j}}{m-j}\left|1-\frac{j}{m}-\frac{j}{m n}\right|\right)\left|B_{n}\left(e_{j} ; z\right)\right|+\frac{n^{m-1}}{(n+1)^{m}} r^{m} \\
\leq & \frac{2 m(n+1)^{m-1}+(n+1)^{m-1}}{(n+1)^{m}} r^{m} \\
= & \frac{(n+1)^{m-1}(2 m+1)}{(n+1)^{m}} r^{m}=\frac{(2 m+1)}{(n+1)} r^{m} .
\end{aligned}
$$

It is well known that, by a linear transformation, the Bernstein inequality in the closed unit disk becomes

$$
\left|P_{m}^{\prime}(z)\right| \leq \frac{m}{r}\left\|P_{m}\right\|_{r}, \quad \text { for all }|z| \leq r, r \geq 1
$$

for all $|z| \leq r$, where $P_{m}(z)$ is a complex polynomial of degree $\leq m$. From the above recurrence formula (12) we get

$$
\begin{aligned}
\left|K_{n}\left(e_{m} ; z\right)-e_{m}(z)\right| & \leq \frac{|z||1-z|}{n}\left|K_{n}^{\prime}\left(e_{m-1} ; z\right)\right|+|z|\left|K_{n}\left(e_{m-1} ; z\right)-e_{m-1}(z)\right|+\frac{(2 m+1)}{(n+1)} r^{m} \\
& \leq \frac{r(1+r)}{n} \frac{m-1}{r}\left\|K_{n}\left(e_{m-1}\right)\right\|_{r}+r\left|K_{n}\left(e_{m-1} ; z\right)-e_{m-1}(z)\right|+\frac{(2 m+1)}{(n+1)} r^{m} \\
& \leq r\left|K_{n}\left(e_{m-1} ; z\right)-e_{m-1}(z)\right|+\frac{2(m-1)}{n} r^{m}+\frac{(2 m+1)}{(n+1)} r^{m} \\
& \leq r\left|K_{n}\left(e_{m-1} ; z\right)-e_{m-1}(z)\right|+\frac{4 m}{n} r^{m} .
\end{aligned}
$$

By writing the last inequality for $m=1,2, \ldots$, we can easily obtain, step by step, the following:

$$
\begin{aligned}
& \left|K_{n}\left(e_{m} ; z\right)-e_{m}(z)\right| \\
& \quad \leq \frac{4 m}{n} r^{m}+r \frac{4(m-1)}{n} r^{m-1}+r^{2} \frac{4(m-2)}{n} r^{m-2}+\cdots+r^{m-1} \frac{4}{n} r \\
& \quad=\frac{4}{n} r^{m}(m+m-1+\cdots+1) \leq \frac{2 m(m+1)}{n} r^{m} .
\end{aligned}
$$

Since $K_{n, q}(f ; z)$ is analytic in $\mathbb{D}_{R}($ see $[7$, p.6]), we can write

$$
K_{n}(f ; z)=\sum_{m=0}^{\infty} a_{m} K_{n}\left(e_{m} ; z\right), \quad z \in \mathbb{D}_{R}
$$


which together with (13) immediately implies for all $|z| \leq r$

$$
\left|K_{n}(f ; z)-f(z)\right| \leq \sum_{m=0}^{\infty}\left|a_{m}\right|\left|K_{n}\left(e_{m} ; z\right)-e_{m}(z)\right| \leq \frac{2}{n} \sum_{m=1}^{\infty}\left|c_{m}\right| m(m+1) r^{m} .
$$

Proof of Theorem 2 A simple calculation and the use of the recurrence formula (10) lead us to the following relationship:

$$
\begin{aligned}
E_{n, m}(z)= & \frac{z(1-z)}{n}\left(K_{n, q}\left(e_{m-1} ; z\right)-e_{m-1}(z)\right)^{\prime}+z E_{n, m-1}(z)+\frac{m-1}{n(n+1)} z^{m-1}(1-z) \\
& +\frac{1}{n+1}\left(z^{m}-B_{n}\left(e_{m} ; z\right)\right)+\frac{1}{n+1}\left(1-\frac{n^{m-1}}{(n+1)^{m-1}}\right) B_{n}\left(e_{m} ; z\right) \\
& +\frac{(\alpha+1)}{(\alpha+\beta+2)(n+1)}\left(\frac{n^{m-1}}{(n+1)^{m-1}}-1\right) B_{n}\left(e_{m-1} ; z\right) \\
& +\frac{\alpha+1}{(\alpha+\beta+2)(n+1)}\left(B_{n}\left(e_{m-1} ; z\right)-z^{m-1}\right)-\frac{(m-1) n^{m-2}}{(\alpha+\beta+2)(n+1)^{m}} B_{n}\left(e_{m-1} ; z\right) \\
& +\frac{1}{(n+1)^{m}} \sum_{j=0}^{m-2}\left(\begin{array}{c}
m \\
j
\end{array}\right) \frac{n^{j} B(\alpha+m-j+1, \beta+1)}{B n-j(n+1)} \\
B n+1, \beta+1) & B_{n}\left(e_{j} ; z\right) \\
:= & \sum_{k=1}^{9} I_{k} .
\end{aligned}
$$

Firstly we estimate $I_{3}, I_{8}$. It is clear that

$$
\begin{aligned}
& \left|I_{3}\right| \leq \frac{m-1}{n(n+1)} r^{m-1}(1+r), \\
& \left|I_{8}\right| \leq \frac{(m-1)}{(\alpha+\beta+2)(n+1)^{2}}\left|B_{n, q}\left(e_{m-1} ; z\right)\right| \leq \frac{(m-1)}{(\alpha+\beta+2)(n+1)^{2}} r^{m-1} .
\end{aligned}
$$

Secondly using the known inequality

$$
1-\prod_{k=1}^{m} x_{k} \leq \sum_{k=1}^{m}\left(1-x_{k}\right), \quad 0 \leq x_{k} \leq 1
$$

to estimate $I_{5}, I_{6}, I_{9}$, we have

$$
\begin{aligned}
\left|I_{5}\right| \leq & \frac{1}{n+1}\left(1-\frac{n^{m-1}}{(n+1)^{m-1}}\right)\left|B_{n}\left(e_{m} ; z\right)\right| \leq \frac{m-1}{(n+1)^{2}} r^{m}, \\
\left|I_{6}\right| \leq & \frac{\alpha+1}{(\alpha+\beta+2)(n+1)}\left(1-\frac{n^{m-1}}{(n+1)^{m-1}}\right)\left|B_{n}\left(e_{m-1} ; z\right)\right| \leq \frac{(m-1)}{(n+1)^{2}} r^{m-1}, \\
\left|I_{9}\right| \leq & \frac{1}{(n+1)^{m}} \sum_{j=0}^{m-2}\left(\begin{array}{c}
m-2 \\
j
\end{array}\right) \frac{m(m-1)}{(m-j)(m-j-1)} \\
& \times \frac{n^{j} B(\alpha+m-j+1, \beta+1)}{B(\alpha+1, \beta+1)}\left(1-\frac{j}{m}-\frac{j}{m n}\right) r^{j} \\
\leq & \frac{2 m(m-1)(\alpha+1)(\alpha+2)(n+1)^{m-2}}{(\alpha+\beta+2)(\alpha+\beta+3)(n+1)^{m}} r^{m} \leq \frac{2 m(m-1)}{(n+1)^{2}} r^{m} .
\end{aligned}
$$


Finally we estimate $I_{4}, I_{7}$. We use [7, Theorem 1.5 .1$]$ :

$$
\begin{aligned}
\left|I_{4}\right|+\left|I_{7}\right| & \leq \frac{1}{n+1}\left|z^{m}-B_{n}\left(e_{m} ; z\right)\right|+\frac{1}{(\alpha+\beta+2)(n+1)}\left|B_{n}\left(e_{m-1} ; z\right)-z^{m-1}\right| \\
& \leq \frac{3 m(m-1)}{2 n(n+1)}(1+r) r^{m-1}+\frac{3(m-1)(m-2)}{2(\alpha+\beta+2) n(n+1)}(1+r) r^{m-2} .
\end{aligned}
$$

Using (13), (15), (16), and (17) in (14) finally we have $(m \geq 3)$

$$
\begin{aligned}
& \left|E_{n, m}(z)\right| \\
& \leq \frac{r(1+r)}{n}\left|\left(K_{n}\left(e_{m-1} ; z\right)-e_{m-1}(z)\right)^{\prime}\right|+r\left|E_{n, m-1}(z)\right|+\frac{m-1}{n(n+1)} r^{m-1}(1+r) \\
& +\frac{3 m(m-1)}{2 n(n+1)}(1+r) r^{m-1}+\frac{m-1}{(n+1)^{2}} r^{m}+\frac{(m-1)}{(n+1)^{2}} r^{m-1} \\
& +\frac{3(m-1)(m-2)}{2(\alpha+\beta+2) n(n+1)}(1+r) r^{m-2}+\frac{(m-1)}{(\alpha+\beta+2)(n+1)^{2}} r^{m-1}+\frac{2 m(m-1)}{(n+1)^{2}} r^{m} \\
& \leq \frac{r(1+r)}{n}\left|\left(K_{n}\left(e_{m-1} ; z\right)-e_{m-1}(z)\right)^{\prime}\right|+r\left|E_{n, m-1}(z)\right| \\
& +\frac{2(m-1)}{n^{2}} r^{m}+\frac{3 m(m-1)}{n^{2}} r^{m}+\frac{m-1}{n^{2}} r^{m}+\frac{(m-1)}{n^{2}} r^{m}+\frac{3(m-1)(m-2)}{(\alpha+\beta+2) n^{2}} r^{m} \\
& +\frac{(m-1)}{(\alpha+\beta+2) n^{2}} r^{m}+\frac{2 m(m-1)}{n^{2}} r^{m} \\
& \leq \frac{r(1+r)}{n}\left|\left(K_{n}\left(e_{m-1} ; z\right)-e_{m-1}(z)\right)^{\prime}\right|+r\left|E_{n, m-1}(z)\right| \\
& +\frac{2 m(m-1)}{n^{2}} r^{m}+\frac{3 m(m-1)}{n^{2}} r^{m}+\frac{m(m-1)}{n^{2}} r^{m}+\frac{m(m-1)}{n^{2}} r^{m} \\
& +\frac{3(m-1)(m-2)}{(\alpha+\beta+2) n^{2}} r^{m}+\frac{m(m-1)}{(\alpha+\beta+2) n^{2}} r^{m}+\frac{2 m(m-1)}{n^{2}} r^{m} \\
& \leq \frac{r(1+r)}{n}\left|\left(K_{n}\left(e_{m-1} ; z\right)-e_{m-1}(z)\right)^{\prime}\right|+r\left|E_{n, m-1}(z)\right| \\
& +\frac{9 m(m-1)}{n^{2}} r^{m}+\frac{4 m(m-1)}{(\alpha+\beta+2) n^{2}} r^{m} \\
& \leq \frac{r(1+r)}{n} \frac{m-1}{r}\left\|K_{n}\left(e_{m-1} ; z\right)-e_{m-1}(z)\right\|_{r}+r\left|E_{n, m-1}(z)\right| \\
& +\frac{9 m(m-1)}{n^{2}} r^{m}+\frac{4 m(m-1)}{(\alpha+\beta+2) n^{2}} r^{m} \\
& \leq \frac{(m-1)(1+r)}{n} \frac{2(m-1) m}{n} r^{m-1}+r\left|E_{n, m-1}(z)\right|+\frac{9 m(m-1)}{n^{2}} r^{m}+\frac{4 m(m-1)}{(\alpha+\beta+2) n^{2}} r^{m} \\
& \leq r\left|E_{n, m-1}(z)\right|+\frac{4 m(m-1)^{2}}{n^{2}} r^{m}+\frac{9 m(m-1)}{n^{2}} r^{m}+\frac{4 m(m-1)}{(\alpha+\beta+2) n^{2}} r^{m} \\
& \leq r\left|E_{n, m-1}(z)\right|+\frac{13 m(m-1)^{2}}{n^{2}} r^{m}+\frac{4 m(m-1)}{(\alpha+\beta+2) n^{2}} r^{m} \text {. }
\end{aligned}
$$

As a consequence, we get

$$
\left|E_{n, m}(z)\right| \leq \frac{13 m(m-1)^{2}}{n^{2}} r^{m}+\frac{4 m(m-1)}{(\alpha+\beta+2) n^{2}} r^{m} .
$$


The result follows from the fact that

$$
\begin{aligned}
& \left|K_{n}(f ; z)-f(z)-\frac{(\alpha+1)-z(\alpha+\beta+2)}{(\alpha+\beta+2)(n+1)} f^{\prime}(z)-\frac{z(1-z)}{2(n+1)} f^{\prime \prime}(z)\right| \\
& \quad \leq \sum_{m=2}^{\infty}\left|a_{m}\right|\left|E_{n, m}(z)\right| \\
& \quad \leq \frac{13}{n^{2}} \sum_{m=2}^{\infty}\left|a_{m}\right| m(m-1)^{2} r^{m}+\frac{4}{(\alpha+\beta+2) n^{2}} \sum_{m=3}^{\infty}\left|a_{m}\right| m(m-1) r^{m} .
\end{aligned}
$$

Note that since $f^{(3)}=\sum_{m=4}^{\infty} a_{m} m(m-1)(m-2) z^{m-3}$ and the series is absolutely convergent for all $|z|<R$, the finiteness of the involved constants in the statement easily follows.

Proof of Theorem 3 For all $z \in \mathbb{D}_{R}$ and $n \in \mathbb{N}$ we get

$$
\begin{aligned}
K_{n, q}(f ; z)-f(z) & \\
= & \frac{1}{n+1}\left\{\frac{(\alpha+1)-z(\alpha+\beta+2)}{(\alpha+\beta+2)} f^{\prime}(z)+\frac{z(1-z)}{2} f^{\prime \prime}(z)\right. \\
& \left.\quad+(n+1)\left(K_{n, q}(f ; z)-f(z)-\frac{(\alpha+1)-z(\alpha+\beta+2)}{(\alpha+\beta+2)} f^{\prime}(z) m z^{m-1}-\frac{z(1-z)}{2(n+1)} f^{\prime \prime}(z)\right)\right\} .
\end{aligned}
$$

We apply

$$
\|F+G\|_{r} \geq\left|\|F\|_{r}-\|G\|_{r}\right| \geq\|F\|_{r}-\|G\|_{r}
$$

to get

$$
\begin{aligned}
& \left\|K_{n, q}(f)-f\right\|_{r} \\
& \geq \frac{1}{n+1}\left\{\left\|\frac{(\alpha+1)-z(\alpha+\beta+2)}{(\alpha+\beta+2)} f^{\prime}(z)+\frac{z(1-z)}{2} f^{\prime \prime}(z)\right\|_{r}\right. \\
& \left.-(n+1)\left\|K_{n}(f ; z)-f(z)-\frac{(\alpha+1)-z(\alpha+\beta+2)}{(\alpha+\beta+2)} f^{\prime}(z)-\frac{z(1-z)}{2(n+1)} f^{\prime \prime}(z)\right\|_{r}\right\} \text {. }
\end{aligned}
$$

Because by hypothesis $f$ is not a constant in $\mathbb{D}_{R}$, it follows

$$
\left\|\frac{(\alpha+1)-z(\alpha+\beta+2)}{(\alpha+\beta+2)} m z^{m-1}+\frac{z(1-z)}{2} m(m-1) z^{m-2}\right\|_{r}>0 .
$$

Indeed, assuming the contrary it follows that $\frac{(\alpha+1)-z(\alpha+\beta+2)}{(\alpha+\beta+2)} m z^{m-1}+\frac{z(1-z)}{2} m(m-1) z^{m-2}=0$, for all $z \in \overline{\mathbb{D}}_{R}$, that is,

$$
\begin{aligned}
& \sum_{m=1}^{\infty} a_{m}\left(\frac{(\alpha+1)}{(\alpha+\beta+2)}-z\right) m z^{m-1}+\sum_{m=2}^{\infty} a_{m} \frac{z(1-z)}{2} m(m-1) z^{m-2}=0 \\
& \sum_{m=1}^{\infty} a_{m} \frac{(\alpha+1)}{(\alpha+\beta+2)} m z^{m-1}-\sum_{m=1}^{\infty} a_{m} m z^{m}+\frac{1}{2} \sum_{m=2}^{\infty} a_{m} m(m-1) z^{m-1}
\end{aligned}
$$




$$
\begin{aligned}
& -\frac{1}{2} \sum_{m=2}^{\infty} a_{m} m(m-1) z^{m}=0 \\
& 0=\frac{(\alpha+1)}{(\alpha+\beta+2)} a_{1}+\sum_{m=1}^{\infty} a_{m+1} \frac{(\alpha+1)}{(\alpha+\beta+2)}(m+1) z^{m}-\sum_{m=1}^{\infty} a_{m} m z^{m} \\
& \quad+\frac{1}{2} \sum_{m=1}^{\infty} a_{m+1}(m+1)(m) z^{m}-\frac{1}{2} \sum_{m=2}^{\infty} a_{m} m(m-1) z^{m} \\
& \frac{(\alpha+1)}{(\alpha+\beta+2)} a_{1}+\sum_{m=1}^{\infty}\left(a_{m+1} \frac{(\alpha+1)}{(\alpha+\beta+2)}(m+1)-a_{m} m\right. \\
& \left.+\frac{1}{2} a_{m+1}(m+1)(m)-\frac{1}{2} a_{m} m(m-1)\right) z^{m}=0, \\
& a_{1}=0, \\
& a_{m+1} \frac{(\alpha+1)}{(\alpha+\beta+2)}(m+1)-a_{m} m+\frac{1}{2} a_{m+1}(m+1)(m)-\frac{1}{2} a_{m} m(m-1)=0 \\
& a_{m+1}\left(\frac{(\alpha+1)}{(\alpha+\beta+2)}(m+1)+\frac{1}{2}(m+1)(m)\right)=a_{m}\left(m+\frac{1}{2} m(m-1)\right) \\
& a_{m+1}=\frac{a_{m}\left(m+\frac{1}{2} m(m-1)\right)}{(\alpha+\beta+2)}(m+1)+\frac{1}{2}(m+1)(m)
\end{aligned}
$$

for all $z \in \overline{\mathbb{D}}_{R} \backslash\{0\}$. Thus $a_{m}=0, m=1,2,3, \ldots$ Thus, $f$ is constant, which is in contradiction with the hypothesis.

Now, by Theorem 2 we have

$$
\begin{aligned}
& (n+1)\left|K_{n, q}(f ; z)-f(z)-\frac{(\alpha+1)-z(\alpha+\beta+2)}{(\alpha+\beta+2)} m z^{m-1}-\frac{z(1-z)}{2} m(m-1) z^{m-2}\right| \\
& \quad \leq \frac{n+1}{n}\left(\frac{13}{n} \sum_{m=2}^{\infty}\left|a_{m}\right| m^{2}(m-1)^{2} r^{m}+\frac{4}{(\alpha+\beta+2) n} \sum_{m=2}^{\infty}\left|a_{m}\right|(m-1)(m-2) r^{m}\right) \rightarrow 0 \\
& \quad \text { as } n \rightarrow \infty
\end{aligned}
$$

Consequently, there exists $n_{1}$ (depending only on $f$ and $r$ ) such that, for all $n \geq n_{1}$, we have

$$
\begin{aligned}
& \left\|\frac{(\alpha+1)-z(\alpha+\beta+2)}{(\alpha+\beta+2)} m z^{m-1}+\frac{z(1-z)}{2} m(m-1) z^{m-2}\right\|_{r} \\
& \quad-(n+1) \| K_{n}(f ; z)-f(z)-\frac{1}{(n+1)}\left(\frac{(\alpha+1)-z(\alpha+\beta+2)}{(\alpha+\beta+2)} m z^{m-1}\right. \\
& \left.\quad+\frac{z(1-z)}{2} m(m-1) z^{m-2}\right) \|_{r} \\
& \geq \frac{1}{2}\left\|\frac{(\alpha+1)-z(\alpha+\beta+2)}{(\alpha+\beta+2)} m z^{m-1}+\frac{z(1-z)}{2} m(m-1) z^{m-2}\right\|_{r}
\end{aligned}
$$

which implies

$$
\left\|K_{n}(f)-f\right\|_{r} \geq \frac{1}{n+1} \frac{1}{2}\left\|\frac{(\alpha+1)-z(\alpha+\beta+2)}{(\alpha+\beta+2)} m z^{m-1}+\frac{z(1-z)}{2} m(m-1) z^{m-2}\right\|_{r}
$$

for all $n \geq n_{1}$. 
For $1 \leq n \leq n_{1}-1$ we have

$$
\left\|K_{n}(f)-f\right\|_{r} \geq \frac{1}{2(n+1)}\left((n+1)\left\|K_{n}(f)-f\right\|_{r}\right)=\frac{1}{2(n+1)} M_{r, n}(f)>0,
$$

which finally implies that

$$
\left\|K_{n}(f)-f\right\|_{r} \geq \frac{1}{n+1} C_{r}(f)
$$

for all $n$, with $C_{r}(f)=\min \left\{\frac{1}{2} M_{r, 1}(f), \ldots, \frac{1}{2} M_{r, n_{1}-1}(f), \frac{1}{2} \| \frac{(\alpha+1)-z(\alpha+\beta+2)}{(\alpha+\beta+2)} m z^{m-1}+\frac{z(1-z)}{2} m(m-\right.$ 1) $\left.z^{m-2} \|_{r}\right\}$.

\section{Competing interests}

The authors declare that they have no competing interests.

\section{Authors' contributions}

All authors contributed equally to the writing of this paper. All authors read and approved the final manuscript.

Received: 13 November 2014 Accepted: 21 January 2015 Published online: 05 February 2015

\section{References}

1. Bernstein, SN: Démonstration du théorème de Weierstrass fondée sur le calcul de probabilités. Commun. Soc. Math. Kharkow 13(2), 1-2 (1912/1913)

2. Ditzian, Z, Totik, V: Moduli of Smoothness. Springer, Berlin (1987)

3. Della Vecchia, B, Mastroianni, G, Szabados, J: A weighted generalization of the classical Kantorovich operator. Rend. Circ. Mat. Palermo Suppl. 82, 1-27 (2010)

4. Della Vecchia, B, Mastroianni, G, Szabados, J: A weighted generalization of the classical Kantorovich operator. II. Saturation. Mediterr. J. Math. 10(1), 1-15 (2013)

5. Yu, DS: Weighted approximation by modified Kantorovich-Bernstein operators. Acta Math. Hung. 141(1-2), 132-149 (2013)

6. Mahmudov, NI, Kara, M: Approximation theorems for generalized complex Kantorovich-type operators. J. Appl. Math 2012, Article ID 454579 (2012)

7. Gal, SG: Approximation by Complex Bernstein and Convolution Type Operators. Series on Concrete and Applicable Mathematics, vol. 8. World Scientific, Singapore (2009)

8. Gal, SG: Overconvergence in Complex Approximation. Springer, New York (2013)

\section{Submit your manuscript to a SpringerOpen ${ }^{\circ}$ journal and benefit from:}

- Convenient online submission

Rigorous peer review

- Immediate publication on acceptance

- Open access: articles freely available online

- High visibility within the field

- Retaining the copyright to your article 